\title{
Questes
}

Revue pluridisciplinaire d'études médiévales

\section{Transmission du pouvoir et transmission du nom : l'exemple de la seigneurie des Trinci de Foligno à la fin du Moyen Âge}

Jean-Baptiste Delzant

\section{OpenEdition}

\section{Journals}

Édition électronique

URL : http://journals.openedition.org/questes/613

DOI : $10.4000 /$ questes.613

ISSN : 2109-9472

Éditeur

Les Amis de Questes

\section{Édition imprimée}

Date de publication : 15 mars 2007

Pagination : 56-66

ISSN : 2102-7188

\section{Référence électronique}

Jean-Baptiste Delzant, «Transmission du pouvoir et transmission du nom : l'exemple de la seigneurie des Trinci de Foligno à la fin du Moyen Âge », Questes [En ligne], 11 | 2007, mis en ligne le 01 janvier 2014, consulté le 20 avril 2019. URL : http://journals.openedition.org/questes/613; DOI : 10.4000/ questes.613 


\section{Transmission du pouvoir et transmission du nom :}

\section{l'exemple de la seigneurie des Trinci de Foligno à la fin du Moyen Âge}

Jean-Baptiste DELZANT

L'histoire politique de l'Italie communale est marquée par une spécificité : le développement de l'indépendance politique de nombreuses cités, qui adoptent un mode de gouvernement fondé sur des décisions collégiales placées sous l'autorité de magistrats élus. Dans un contexte général de tensions sociales et de luttes intestines, une tendance lourde se dégage pourtant, qui voit, dans certains cas dès la seconde moitié du XII ${ }^{\mathrm{e}}$ siècle, les principales fonctions décisionnelles regroupées dans les mains d'un seul homme. Avec la naissance des seigneuries, un siècle plus tard, le pouvoir est accaparé, alors que les institutions communales sont progressivement vidées de tout contenu ${ }^{1}$.

Afin de justifier leur position et la patrimonialisation du pouvoir, les seigneurs élaborent une véritable propagande, autour du thème central de la capacité à assurer la continuité du gouvernement de la cité et à transmettre le pouvoir sans heurt. Ce discours s'avère fragile, car en droit, la direction des affaires de la cité est exercée au nom d'une délégation temporaire du pouvoir par la Commune ou par une instance extérieure, le pape ou l'empereur. Le pouvoir est une charge, concédée ou déléguée, non un titre, propriété

1 Élisabeth Crouzet-PAVAn, Enfers et paradis. L'Italie de Dante et de Giotto, Paris, Albin Michel, 2001, p. 244-252. 
personnelle et transmissible ${ }^{2}$. Le discours seigneurial doit dès lors légitimer la conservation et la transmission du pouvoir à l'intérieur d'une même famille.

Ce discours est étudié ici à travers l'exemple de la famille des Trinci, qui dominent de 1305 à 1439, de façon quasi continue, la petite cité ombrienne de Foligno $^{3}$. Pour ces seigneurs, le choix et la transmission du nom fonctionnent comme un principe identitaire délimitant et renforçant un groupe familial à l'intérieur duquel le pouvoir peut être exercé ${ }^{4}$. Deux moments viennent étayer cette démarche. L'adoption d'un système de dénomination permet tout d'abord de créer et de mettre en avant une lignée dont la continuité justifie l'exercice du pouvoir. Le nom n'est cependant pas un simple marqueur social, il est également création d'un imaginaire dans lequel les origines mythiques garantissent la légitimité de la transmission.

Quelques jalons d'une étude anthroponymique portant sur la façon dont s'est constitué le nom des Trinci peuvent être proposés : l'onomastique révèle l'importance de la préoccupation dynastique chez cette famille. La mise en place du système de dénomination intervient au moment où il importait aux Trinci de construire une véritable lignée dans laquelle inscrire leur récent pouvoir seigneurial. La cité de Foligno est le théâtre d'un violent conflit opposant deux familles, les Trinci et les Anastasi, pour le contrôle des institutions communales. En 1305, le chef des premiers, Nallo, aidé de ses voisins pérugins, chasse son

\footnotetext{
${ }^{2}$ Giovanni TABACCO, «L'Italia delle signorie », dans Signorie in Umbria tra Medioevo e Rinascimento :l'esperienza dei Trinci, Congresso storico internazionale, Foligno, 10-13 décembre 1986, Pérouse, Deputazione di Storia Patria per l'Umbria, 1989, p. 9-10.

${ }^{3}$ Ils y ont laissé un superbe palais, principalement édifié sous la seigneurie d'Ugolino III (entre 1386 et 1415) et décoré à fresques au cours de campagnes successives. L'une d'elles, menée dans la seconde moitié des années 1400, a été placée sous la direction de Gentile da Fabriano.

${ }^{4}$ L'article de François Menant propose un excellent exposé sur les questions identitaires liées au système de dénomination au Moyen Âge. Voir François MENANT, «Entre la famille et l'État: l'héritage du nom et ses détours dans l'Italie des communes », dans J.-M. MARTIN et François MENANT (éds.), Genèse médiévale de l'anthroponymie moderne : l'espace italien. Mélanges de l'École française de Rome, Moyen Âge, 110 (1998), p. 253-270.
} 
rival. Bonaventura di Benvenuto, chroniqueur local, raconte ainsi la prise de pouvoir :

[...] item die penultima iunii potestas et milites Perusini venerunt et hospitati fuerunt Fulginei, et die prima iulii de mane intraverunt plateam veterem et multi Fulginates secuti sunt eos, et populus elegit in capitaneum populi Nallum domini Trincie, et ceperunt palatium populi cum turre, et ex eo expulerunt consules populi circa tertiam, et tunc Conradus Anestasii exivit civitatem et ivit Tudertum. ${ }^{5}$

Lorsque Bonaventura écrit, vers le milieu du $\mathrm{XIV}^{\mathrm{e}}$ siècle, c'est tout naturellement qu'il désigne le premier seigneur par le nom de «Trincie». Les quelques éléments d'archives mis à jour faisant référence aux membres du lignage avant le coup de force de 1305 attestent toutefois l'existence d'un autre système de dénomination. Celui-ci, majoritairement répandu dans l'Italie du temps, repose sur la reprise, par le fils, du nom du père (nomen paternum). Après plusieurs générations, les noms sont utilisés les uns à la suite des autres, aboutissant à une chaîne de nomina paterna. Les archives de l'abbaye de Sassovivo renfermaient des actes impliquant le grand-père de Nallo, désigné de la façon suivante : Trincia di Berardo di Rodolfo ${ }^{6}$, c'est-à-dire Trincia, fils de Berardo, lui-même fils de Rodolfo. Trincia eut un fils, lui aussi prénommé Trincia, et qui mit son épée au service de Frédéric II. Grâce à cet engagement, il put constituer un patrimoine foncier considérable. Le nom de famille des seigneurs de Foligno provient ainsi du père du premier seigneur. La reprise et la transmission du nomen paternum aux générations successives sous forme de

\footnotetext{
${ }^{5}$ «L'avant dernier jour de juin, le podestat et les cavaliers de Pérouse arrivèrent et furent introduits dans Foligno. Le premier jour de juillet, ils entrèrent sur la vieille place, et de nombreux Folignats se joignirent à eux : le peuple élit le seigneur Nallo Trinci capitaine du Popolo. La foule s'empara du palais et de ses tours, et vers tierce, en chassa les consuls du Peuple. Alors Corrado Anastasi s'enfuit et gagna Todi ». B. di Benvenuto, Cronaca, dans Michele Faloci Pulignani, Framgenta fulginatis historiae. Rerum Italicarum Scriptores, Bologne, N. Zanichelli, tome 26, Partie II, 1932-33. Édition consultée dans le tirage du fascicule à part 264, 1933, p. 20.

${ }^{6}$ Cité par Mario SENSI, «I Trinci tra storia, storiografia ed erudizione », dans Signorie in Umbria..., op. cit., p. 177.
} 
nom collectif marquent la volonté de se référer à l'homme qui, grâce aux terres acquises et transmises à ses fils, apparaît comme le fondateur de la lignée. Les seigneurs de Foligno suivent en cela les nombreuses familles qui adoptent pour célébrer et renforcer leurs solidarités lignagères un nom collectif héréditaire en de suivi d'un ablatif pluriel. Dans le même fonds de l'abbaye de Sassovivo, deux générations plus tard, le changement s'est bien opéré. Nallo, nouveau maître de la ville, est mentionné dans des documents datant de 1309, 1319 et 1321, en ces termes : nobilis et potens vir Nallus $d$. Trincie $d$. Trincie d. Barardi de Trinciis confalonerius iustitie et capitanus populi civitatis Fulginei ${ }^{7}$. Le nom collectif a été ajouté à la chaîne de nomina paterna ${ }^{8}$. Tout se passe comme si c'était au moment où les Trinci prenaient le pouvoir qu'ils adoptaient comme mode de désignation le nom collectif en de suivi d'un ablatif pluriel, tout à la fois affirmation de fierté et volonté de renforcement lignager.

Le mécanisme décrit peut fonctionner en sens inverse, et le patronyme peut être utilisé comme prénom, Trincia. À moins qu'il ne s'agisse pour les parents de reprendre le prénom d'un ancêtre important. Quel que soit le sens de la démarche, le résultat est le même: Trincia Trinci. Ce phénomène de redoublement ancre encore plus profondément celui qui est ainsi nommé dans l'histoire familiale. En outre, dans le choix de leurs prénoms, les Trinci conservent, dans une attitude aristocratique, un répertoire de noms germaniques, comme Corrado ou Manfredonio. La transmission des prénoms illustre elle aussi

\footnotetext{
${ }^{7}$ Ibid., p. 177.

${ }^{8}$ Les archives pontificales confirment cette interprétation. La lettre que Boniface VIII envoie aux trois fils de Trincia «le Jeune» en 1296 commence ainsi : Dilectis filiis Corrado, Ugolino et Renaldo [c'est-à-dire Nallo] filiis Trincie de Fulgineo. Dans les lettres écrites ensuite par la chancellerie pontificale aux seigneurs de Foligno, ceux-ci sont nommés par leur prénom suivi du de Trinciis. Les éléments empruntés à deux fonds d'archives différents semblent donc converger. Ces quelques extraits restent malheureusement trop peu nombreux pour pouvoir constituer une véritable étude anthroponymique fondée sur des séries significatives.
} 
de façon particulièrement nette la volonté de continuité dynastique ${ }^{9}$. Dans la seule branche régnante, "Corrado » et «Ugolino » sont employés à chaque génération: Nallo, qui s'empare du pouvoir en 1305, a deux frères qui portent ces nomina. Il les donne ensuite à deux de ses fils. L'un d'eux, Ugolino II Novello, prénomme ses garçons Trincia et Corrado. Le premier transmet le nomen Ugolino à son fils aîné (il s'agit d'Ugolino III). Cette transmission de prénoms est un processus permanent de construction lignagère. Chaque homme portant le nom de Corrado ou d'Ugolino s'inscrit dans la continuité de ses prédécesseurs et assure la pérennité du nom, et donc du pouvoir, pour la durée de sa vie.

Ces stratégies familiales sont doublées par des efforts politiques visant à s'assurer que la transmission du pouvoir se fasse bien à l'intérieur de la lignée récemment constituée, selon un principe héréditaire établi en droit. L'autorité sur la cité est, formellement, exercée selon le principe de la delegatio, au nom de la Commune. Elle est néanmoins confirmée par une instance supérieure, la papauté. Cette dernière, du fait de son exil avignonnais au cours du XIV siècle, est à la recherche de soutien et de relais politique dans les terres italiennes placées sous son autorité. En lui apportant leur fidélité et leur soutien, les Trinci réussissent à en obtenir une reconnaissance officielle. Ainsi, pour la première fois, en 1367, Trincia se voit octroyer la charge de vicaire apostolique pour une durée de dix ans. Ce n'est qu'avec le Grand Schisme que les Trinci parviennent à garantir la reconduction automatique de leur fonction à leurs descendants. La charge de vicaire apostolique de Foligno conférée en 1392 par Boniface IX est transmissible aux descendants d'Ugolino si celui-ci vient à mourir avant le terme de son vicariat ${ }^{10}$. C'est le même pape qui pour la première fois, en 1398,

\footnotetext{
9 John LAw, «Le prince de la Renaissance », dans Eugenio GARIN (éd.), L'homme de la Renaissance, Paris, Édtions du Seuil, 1990, p. 33 ( $1^{\text {ère }}$ éd. : L'uomo del Rinascimento, RomeBari, Giuseppe Laterza \& Figli, 1988).

${ }^{10}$ Bulle de Boniface IX à Ugolino Trinci, Rome, 17 août 1392. Publiée par Mario SENSI, « I Trinci... », op. cit., Appendice, document 7, p. 208-209.
} 
octroie la charge pour plusieurs générations (dont le nombre reste fixé) et non plus pour une durée déterminée en années. Avec l'introduction des générations dans la succession héréditaire, le nombre d'années de la concession ne peut plus être prévu. La papauté accroît l'autonomie des Trinci en ce qu'elle les décharge des démarches visant à obtenir la réattribution régulière de leur office. $\mathrm{Ce}$ moment constitue les « prémisses de la construction d'un véritable principat que cependant ni Ugolino ni ses successeurs ne parviennent à concrétiser ${ }^{11}$.

Pour que la transmission héréditaire du pouvoir soit assurée de façon pérenne au sein d'une lignée, les seigneurs ne peuvent se contenter d'un processus continu de création dynastique ou de l'obtention de garanties du maintien de leurs charges pour les générations à venir. L'efficacité de la succession dynastique est également liée à un passé qui doit légitimer le présent et les ambitions futures.

Il faut enraciner la lignée dans un passé lointain et glorieux. La propagande seigneuriale s'efforce d'exalter les prédécesseurs ou de les créer si besoin est, ce qui lui permet de proposer une légitimité antérieure à la delegatio populaire et à la concession pontificale ou impériale ${ }^{12}$. Il s'agit là d'un phénomène récurrent dans des seigneuries qui cherchent à mettre en avant « le rattachement idéal du prince et de sa descendance à un passé mythologique, capable d'affranchir la dynastie de ses véritables origines vassaliques ou communales $»^{13}$. C'est ainsi qu'à Città di Castello, les Vitelli affirment descendre de l'empereur Vitellius (15-69). Ces origines mythiques reposent bien souvent sur une étymologie homophonique, courante dans un Moyen Âge pour lequel les noms des personnes et des objets traduisent quelque chose de la réalité

11 Mario SENSI, «I Trinci... », op. cit., p. 186 : «le premesse per la costituzione di un principato che tuttavia né Ugolino né i suoi successori riuscirono a realizzare ».

12 Sergio Bertelli, «La corte italiana del Quattrocento », dans Federico ZERI (éd.), La pittura in Italia. Il Quattrocento, volume 2, Milan, Electa, 1987, p. 502.

${ }^{13} \mathrm{Ibid}$ : : « il riallaccio ideale del principe e della sua stirpe con un passato mitologoco, capace di staccare la dinastia dalle sue vere origini vassallatiche e/o communali ». 
de leur être et de leur parenté. L'ancêtre est choisi en fonction de la ressemblance que présente la graphie de son nom avec celle du nom de la famille. Cette ressemblance révèle la filiation.

Dans le répertoire des ancêtres illustres, les Trinci choisissent Tros. À la charnière des $\mathrm{XIV}^{\mathrm{e}}$ et $\mathrm{XV}^{\mathrm{e}}$ siècles, Federico Frezzi, dominicain nommé évêque de Foligno peu après la rédaction de son chef-d'œuvre, le Quadriregio, se fait l'écho du mythe familial et exalte la famille régnante. Tros devient le fondateur de Foligno, ce qui permet à Frezzi d'établir un lien organique entre la cité et ses dynastes :

E questos Tros poi in quel tempo vecchio [...]

Flamminea chiamò la città bella, [...]

La città il nome e '1 loco mutò anco ;

E fo Flamminea Foligno nomata [...]. ${ }^{14}$

Un peu plus loin, Frezzi poursuit :

Da questos Tros vien la progenie degna

De' troian Trinci, ed indi è casa Trincia,

Che anco ivi dimora e ivi regna. ${ }^{15}$

Le lettré donne aux seigneurs de Foligno la légitimité qu'ils recherchent: le règne de la famille a commencé avant l'ère chrétienne et l'âge communal. Leur pouvoir se trouve nimbé de l'aura des Troyens.

Le nom de Tros peut évoquer plusieurs personnages, tous liés à la fondation de Troie, et son histoire n'est pas, de la part de Frezzi, une simple flagornerie destinée à attirer les bonnes grâces d'un petit seigneur. Elle semble

14 «t le dit Tros, en ce temps reculé $[\ldots]$

Nomma la belle cité Flamminea [...]

Le nom de la ville et de la région changea de nouveau

Et de Flamminea devint Foligno ».

Federico FrezZI, Il Quadriregio, I, XVIII, édité par E. FILIPPINI, Bari, Gius. Laterza \& Figli, 1914, p. 92-93.

15 «C'est de ce Tros que provient la digne descendance

De Trinci de Troie, et de là, la maison des Trinci

Qui ici même demeure et ici règne ».

Federico FREZZI, op. cit., p. 93. 
faire partie intégrante de l'histoire familiale, comme l'illustre, hors de la branche régnante, l'usage d'un prénom par lequel les Trinci font revivre une histoire mythique dont ils sont les continuateurs. L'un des fils de Corrado I, Rinaldo, change de nom lorsqu'il entre au monastère bénédictin de Santa Croce de Sassovivo en 1381 : il demande à être appelé Troiano ${ }^{16}$.

La relation entre les Trinci et Troie est développée par la décoration du palais, qui y associe un second point de référence, Rome, et enrichit le message dynastique et politique. La loggia présente en effet l'histoire de Romulus et Remus, tout en soulignant les origines troyennes de la cité éternelle. La scène centrale du cycle représente Rhéa Sylvia enterrée vive par son oncle Amulius ${ }^{17}$. Celui-ci a fait emprisonner son propre frère Numitor pour prendre sa place sur le trône d'Albe. Les scènes sont peintes sur les trois murs de la loggia, et le choix qui a présidé à la représentation de chacune, tout comme l'espace qui leur est accordé, est significatif. Tout le mur de gauche est dévolu à Rhéa Sylvia, et l'enterrement de la vestale est placé sous une niche en plein cintre qui encadre la scène et occupe à peine moins de la moitié de la surface dédiée à la narration sur le mur central. Dans l'état actuel des fresques, il s'agit de la scène principale, mise en valeur par sa position dans le cycle, son ampleur, et son insertion dans l'espace et l'architecture réels ${ }^{18}$. La mère de Romulus se voit donc consacrer une importance singulière dans un cycle supposé figurer l'histoire de la fondation de Rome.

L'accent mis sur l'histoire de Rhéa Sylvia crée un effet de résonance entre l'histoire mythique et celle, récente, des Trinci. Le maître de la ville a été renversé par un usurpateur, un meurtre ignoble a été commis, mais les héritiers

\footnotetext{
${ }^{16}$ Maria Virginia ProsPeri VALENTI, «Due Trinci podestà di Firenze nel XIV secolo », dans Bollettino storico della città di Foligno, 2 (1978), p. 130.

${ }^{17}$ Voir illustration à la fin de l'article, p. 66.

${ }^{18}$ Environ un tiers de la surface peinte est perdu, sans même que les sujets choisis soient connus. Cela rend hasardeuse toute interprétation iconographique, comme celle qui propose d'interpréter l'absence du meurtre de Remus comme une volonté de dissimuler les conflits fratricides ou les luttes de faction qui menacent toutes les cités italiennes.
} 
légitimes du pouvoir rétablissent le bon droit et la justice. Ils sont à l'origine d'une nouvelle cité promise à un avenir glorieux ${ }^{19}$. Les fresques ne constituent pas une illustration pure et simple de l'histoire des Trinci, et Romulus et Remus ne sont pas de simples symboles de Corrado $^{20}$ et de son neveu Ugolino, qui règnent alors conjointement sur Foligno. Si l'histoire des seigneurs est bien présente en filigrane, la signification ainsi proposée par une série d'analogies n'épuise pas le sens de l'image. Il ne s'agit pas de peindre, de façon univoque, les Trinci, mais d'établir des conditions de représentation dans lesquelles le spectateur peut de lui-même, sans que l'image le lui impose, penser à des ressemblances. Les analogies sont facilitées par le fait que les personnages de l'Antiquité sont vêtus à la mode médiévale : Rhéa Sylvia porte un vêtement d'un luxe ostentatoire, une robe de pourpre brodée de fils d'or, aux manches longues et échancrées tombant jusqu'au sol. L'insertion, traditionnelle, de détails et de vêtements contemporains dans une scène antique permet une convergence des temporalités. Des niveaux de lecture différents cohabitent dans la fresque sans se superposer ou s'annuler.

Accorder une importance particulière à Rhéa Sylvia accentue avant tout les origines troyennes de Rome. Cette volonté est renforcée par les tituli qui, indissociables du sens produit, explicitent le sujet des fresques ${ }^{21}$. Ils désignent la mère de Romulus et Remus par le nom d'Ilia, et non par celui de Rhéa Sylvia :

Intra Vestali virgini se noma

Ilia nata de re Numitore

\footnotetext{
${ }^{19}$ En 1377, la seigneurie des Trinci est brièvement renversée : la population de Foligno a investi le palais et en a précipité Trincia, le père du commanditaire des fresques, Ugolino III, d'une des fenêtres.

${ }^{20}$ Le frère de Trincia, qui parvient quelques mois après les événements à reprendre le contrôle de la ville.

${ }^{21}$ Les inscriptions murales sont fragmentaires. Le texte est cependant connu grâce à la transcription qui en a été faite au XVII ${ }^{\mathrm{e}}$ siècle par Ludovico Iacobili. Il a été édité par Michele FALOCI PUlignANI, «Le arti e lettere alla corte dei Trinci : ricerche storice », dans Archivio Storico per le Marche e per l'Umbria, 4 (1888), p. 123, ainsi que par Mario SALMI, "Gli affreschi del Palazzo Trinci », dans Bollettino d'Arte, 13, fasc. IX-XII (septembre-décembre 1919), p. 173-174.
} 
La quale de Marte per venereo ardore

Concepì i fondatori dell'alma Roma. ${ }^{22}$

Ilia est pourtant l'épouse d'Ilos, le fils de Tros, qui est également présenté comme le fondateur éponyme d'Ilion, c'est-à-dire de Troie ${ }^{23}$. Nommer la mère de Romulus Ilia, c'est donc convoquer la figure de Tros, et avec elle, de nouveaux parents, les Trinci. Créateurs d'un régime nouveau, ils se donnent pour les refondateurs de Foligno, dont ils ont modifié l'aspect en profondeur par leurs travaux et dont ils ont accru le prestige par leur cour brillante et par d'importantes extensions territoriales. L'image qui se dessine est bien celle de Foligno comme nouvelle Rome. Comme son illustre modèle, Foligno est appelée à un destin glorieux qu'elle peut accomplir sous la conduite des Trinci. La fresque, par les tituli comme par son organisation interne qui met en avant la figure de Rhéa Sylvia, condense ainsi trois références : celle de Romulus et de Rome, celle de Tros et de Troie, celle des Trinci et de Foligno. A travers un jeu d'analogies, elle suscite des associations qui posent l'origine hérö̈que des Trinci et légitime leur pouvoir, grâce à son ancienneté, sa continuité et le caractère exceptionnel de la lignée. Mais l'origine mythologique n'est plus tant un point de départ qu'un élément de comparaison par lequel le continuateur apparaît comme un nouveau fondateur ${ }^{24}$ : ce qui légitime la transmission du pouvoir que le seigneur a reçu, c'est sa capacité à fonder lui-même, comme Romulus, sa propre lignée et sa propre puissance.

\footnotetext{
${ }^{22}$ «L'une des chastes vestales se nommait

Ilia, fille du roi Numitor.

D'un amour ardent avec Mars,

Elle donna naissance aux fondateurs de la noble Rome ».

${ }^{23}$ Comme le remarque Claire VADÉE, « Gli affreschi di Palazzo Trinci e la pittura folignate tra Trecento e Quattrocento ", in Signorie in Umbria..., op. cit., p. 414. L'auteur ne voit cependant dans cette association qu'une confusion due, selon elle, à une tradition littéraire aujourd'hui perdue.

${ }^{24}$ Daniel ARASSE, «Portrait, mémoire familiale et liturgie dynastique : Valerano-Hector au château de Manta », dans Augusto Gentill (éd.), Il ritratto e la memoria. Materiali, Rome, Bulzoni Editore, 1989, note 32, p. 112.
} 


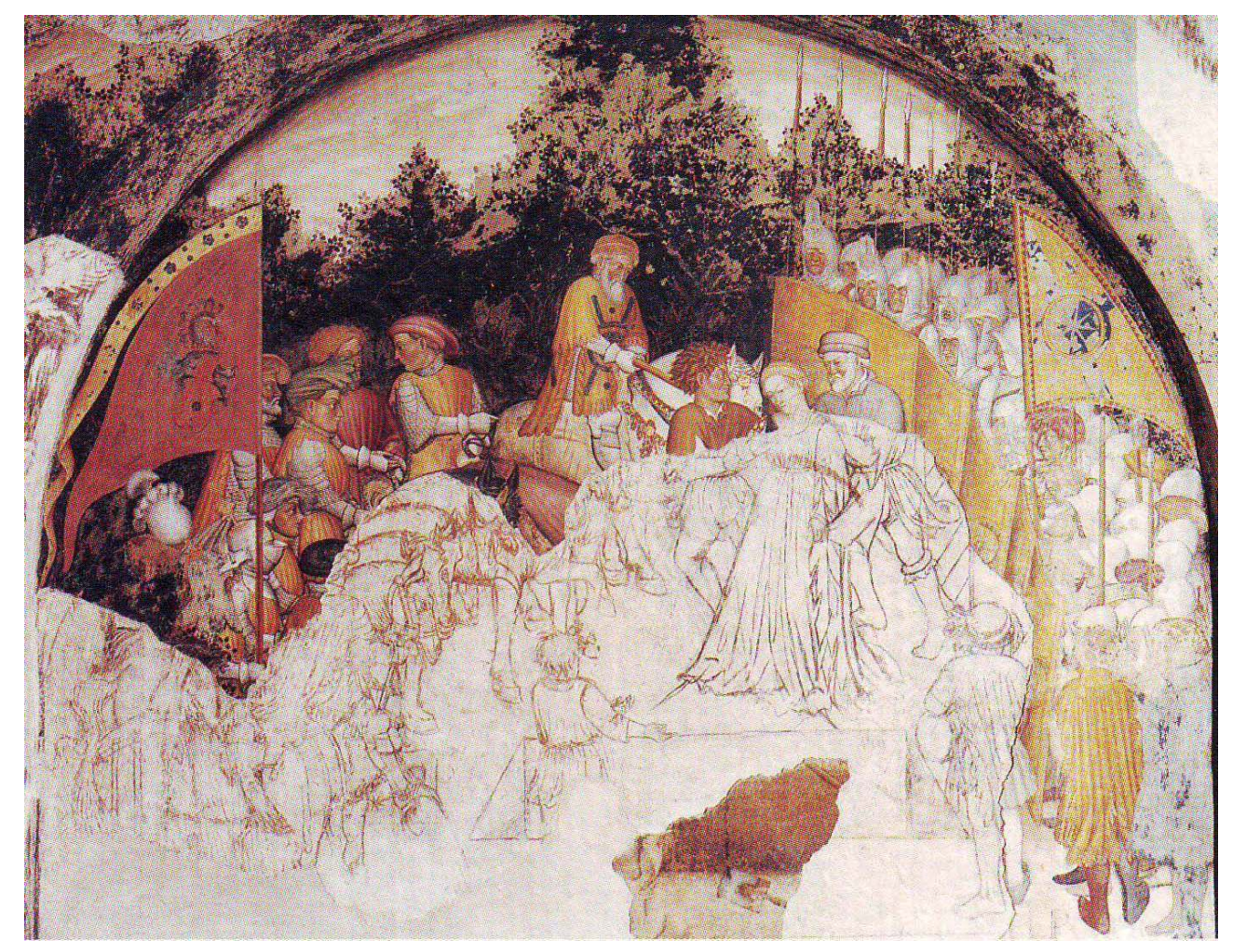

Exécution de Rhéa Sylvia, loggia, palais Trinci, Foligno. Fulginart ( 